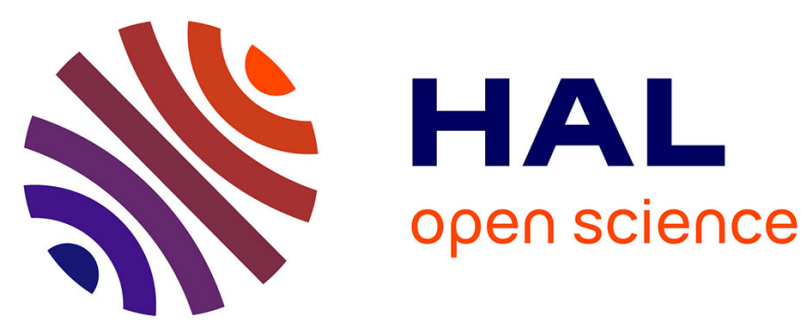

\title{
Numerical modelling of the impact of energy distribution and Marangoni surface tension on track shape in selective laser melting of ceramic material Qiang Chen, Gildas Guillemot, Charles-André Gandin, Michel Bellet
}

\section{- To cite this version:}

Qiang Chen, Gildas Guillemot, Charles-André Gandin, Michel Bellet. Numerical modelling of the impact of energy distribution and Marangoni surface tension on track shape in selective laser melting of ceramic material. Additive Manufacturing, 2018, 21, pp.713 - 723. 10.1016/j.addma.2018.03.003 . hal-01809287

\section{HAL Id: hal-01809287}

https://hal-mines-paristech.archives-ouvertes.fr/hal-01809287

Submitted on 15 Jun 2018

HAL is a multi-disciplinary open access archive for the deposit and dissemination of scientific research documents, whether they are published or not. The documents may come from teaching and research institutions in France or abroad, or from public or private research centers.
L'archive ouverte pluridisciplinaire HAL, est destinée au dépôt et à la diffusion de documents scientifiques de niveau recherche, publiés ou non, émanant des établissements d'enseignement et de recherche français ou étrangers, des laboratoires publics ou privés. 
MINES ParisTech, PSL Research University,

CEMEF - Centre de mise en forme des matériaux, CNRS UMR 7635,

1 rue Claude Daunesse, CS 10207, 06904 Sophia Antipolis Cedex, France

\begin{abstract}
The present study is based on a formerly developed 3D finite element modelling of the selective laser melting process (SLM) at the track scale. This numerical model is used to assess the impact of two phenomena on the shape of the elementary track resulting from SLM processing: laser interaction on one hand, and Marangoni effect on the other hand. As regards laser interaction, it is modelled by a Beer-Lambert type heat source, in which lateral scattering and material absorption are considered through two characteristic parameters. The impact of these parameters is shown in terms of width and depth of melted zone. The Marangoni effect caused by tangential gradients of surface tension is modelled to simulate the fluid dynamics in the melt pool. The resulting convection flow is demonstrated with surface tension values either increasing or decreasing with temperature. The influence of energy distribution, surface tension effects, as well as laser scanning speed on temperature distribution and melt pool geometry is investigated. The stability and regularity of the solidified track are a direct output of the simulations, and their variations with material and process conditions are discussed.
\end{abstract}

Keywords: selective laser melting, laser interaction, surface tension, Marangoni effect, track profile, ceramics

* Corresponding author

Tel.: +33(0)493957461

Email address: michel.bellet@mines-paristech.fr 


\section{Introduction}

In the last decade, Additive Manufacturing (AM) has been largely developed. This innovative technology reflects the current evolution of manufacturing methods in industries. It is sometimes presented as the symbol of the next industrial revolution [1]. Indeed this new technology reduces the time gap between design and manufacturing in order to deliver parts with few finishing work or for direct end-use applications. As parts can be directly printed based on a computer-aided design model, AM processes remove the intermediate costly steps usually required in industries to manufacture pieces. In addition, few constraints are imposed on the geometry of parts, giving the possibility to produce complex shapes that would have required an assembly of several parts with traditional manufacturing technologies.

Different AM processes have been developed in recent years with applications covering a wide range of materials, from polymers to metals. Among them, Selective Laser Melting (SLM) is attractive for the manufacturing of parts requiring high level of mechanical properties, especially in the fields of aerospace and medical orthopedic for metallic alloys hard to shape with conventional cutting, forming or casting technologies. Demonstrations include aluminum [2], stainless steel [3], titanium [4][5], cobalt chromium [6]. With SLM, processed material is totally melted by the use of a focused laser with energy concentrated on a narrow domain. Fully dense parts can thus be achieved and near net shape is possible. However, if the process parameters are not optimized, specific defects like cracks, porosities or poor surface quality may be encountered. All these defects have detrimental consequences on final mechanical properties for direct end-use applications [2]. This justifies efforts made in order to improve the knowledge of physical mechanisms occurring in SLM processes at the local scale as well as the development of associated modelling tools to master and control the formation of elementary tracks. Indeed, an essential difficulty in SLM is to obtain a well attached and fully dense elementary track without cracks. The track should be continuous, stable and with regular shape. Such fine tracks may reduce the formation of porosity and improve the densification of final part [7]. This requires a deep understanding of physical phenomena taking place in SLM.

The specific application of SLM to ceramic materials is basically motivated by the fact that ceramics like alumina or zirconia have outstanding mechanical strength and excellent thermal and wear resistance [8]. However, SLM processing applied on ceramics is known for some difficulties, which explains that industrial applications are not available yet [9]. The first difficulty lies in their low energy absorption to Nd:YAG laser at the wavelength of $1064 \mathrm{~nm}$. This causes high energy loss and reduces production rate. Another difficulty is the formation of cracks in solidified tracks due to the low fracture toughness of ceramics [10][11]. A practical solution of crack elimination was proposed by Hagedorn et al. [8] through preheating the whole part to be just below the melting point. 
As the driving force of the fusion is the laser energy, the interaction between laser and powder is of prime importance. When a laser flux irradiates a powder bed, powder particles are heated by incident radiations. However, in addition to the partial absorption process, radiations can also be reflected and transmitted by powder particles. Multiple reflections between particles usually occur, leading to an irradiated and heated region having a larger diameter than the nominal transverse section of the laser beam and being deeper than in bulk materials [12]. Moreover, the penetration can be quite deep for ceramic materials such as alumina $\left(\mathrm{Al}_{2} \mathrm{O}_{3}\right)$, zirconia $\left(\mathrm{ZrO}_{2}\right)$, or a mix of those two, which can be transparent to the Nd:YAG laser radiation. Consequently, an adequate heat source model should address all these effects in order to propose an efficient modelling of the energy input. The ray tracing method at the powder particle scale was used by Wang and Kruth [13] who found a deeper penetration for highly reflective metallic powder particles. This model was also integrated by Khairallah et al. [14], who pointed out that melting is not uniform and noted more heat accumulation inside powder particles compared with the homogeneous laser deposition. They showed that this effect is induced by low heat extraction through the substrate because of narrow point contact. However, the ray tracing method needs to represent powder particles and to analyze their packing structure, making the simulation complex and time consuming. Gusarov and Smurov [15] described a model assimilating the powder to a homogeneous absorbing and scattering medium. They showed that the radial transport of radiative energy due to scattering in the powder layer can reduce the energy density at the spot center. This phenomenon is more pronounced for narrow laser beams and materials with low absorptivity. This model was later integrated by Hodge et al. [16] and King et al. [17]. Other models like the one based on the Beer-Lambert law can also be found with heterogeneous absorption being taken into account [18][19].

Besides laser/material interaction, the dynamics of melt pool has a significant influence on the shape of the solidified track. Fluid flow in the melt pool is principally driven by external forces like vaporization pressure, surface tension and Marangoni effect [20]. Vaporization often occurs in SLM as the laser energy is concentrated and the vicinity of melt pool under laser spot can easily exceed the boiling temperature. This phenomenon reduces the maximum temperature by taking away a large amount of energy, sometimes spraying out liquid drops [21]. Surface tension has a tendency to smooth the surface of the melt pool inducing different effects on the track shape depending on the scanning speed. At low scanning speed, the melt pool is usually continuous. However, when the velocity increases, the ratio of the melt pool length to its circumference reaches the limit of Plateau-Rayleigh instability [22]. In order to minimize surface energy, the long melt pool is interrupted into fragments, which get spheroidized due to surface tension. This so-called balling effect was observed and analyzed by $\mathrm{Gu}$ and Shen [23], who proposed control methods depending on the 
kind of balling effect. It was reproduced in numerical simulation by Khairallah et al. [14], who pointed out the possibility to control the fluctuation magnitude of melt pool, and thus the balling effect by controlling the heat input over time.

In the present context, an important feature regarding surface tension is that it depends essentially on temperature. That explains the Marangoni effect which is induced by the tangential surface tension gradient along the melt pool surface, where a high tangential temperature gradient is present. This effect is considered to have a marked impact on the melt pool geometry and the temperature distribution inside it [24]. In the context of SLM, Yuan and $\mathrm{Gu}$ [25] developed a 3D finite volume model for TiC/AlSi10Mg nanocomposites considering the Marangoni effect. A wider and shallower melt pool was observed when this effect was taken into account. However, their model does not include the formation of the track shape and consequently the influence of Marangoni effect on its evolution was not investigated. Qiu et al. [26] took into account the surface tension and Marangoni flow with recoil pressure in their model with regularly packed Ti-6Al-4V powder particles. They demonstrated the significant influence of Marangoni force and recoil pressure on the melt splashing and thus the melt pool instability. Khairallah et al. [14] used a fine-scale numerical model with randomly packed $316 \mathrm{~L}$ steel powder particles. They concluded that the Marangoni flow induces the circulation of liquid and hence cools the laser spot region. This flow can also result in liquid spattering away from the surface due to the low liquid metal viscosity.

The authors of the present paper proposed in a previous article [15] a Finite Element (FE) model with a level set formulation dedicated to SLM. This model included a volume heat source based on the Beer-Lambert law as well as an implementation of surface tension. As a consequence, the melting of the powder, the formation of liquid droplets connected to the powder bed as well as the dynamic of the liquid-gas interface upon feeding of the melt pool with liquid droplets could be modeled. However, prediction of both convection and heat flow were limited by the absence of Marangoni convection. This is added in the model presented hereafter and gives access to the prediction of the track height variation, an output of simulations that is rarely available in the literature. In the present paper, the phenomenon of multiple reflections is considered but simplified by a simple enlargement of the Gaussian distribution. The influence of modified source distribution on the temperature field, the melt pool geometry and the shape of solidified track are compared with a non-modified Gaussian distribution. The Marangoni effect (tangential force) due to the surface tension gradient is integrated, in addition to the surface tension in the normal direction. This model is applied to simulate the formation of a single track for pure alumina with different process and material parameters. The resulting convection flow is presented and its role on the homogenization of temperature field is demonstrated. The instability of the melt pool with and without 
Marangoni effect is also investigated, together with their consequences on the melt pool geometry and the final track shape.

\section{Modelling}

The numerical finite element formulation developed to solve the different conservation equations on the simulation domain has been previously detailed by the authors [19]. The following section present the essential features of this model.

\subsection{Level set method}

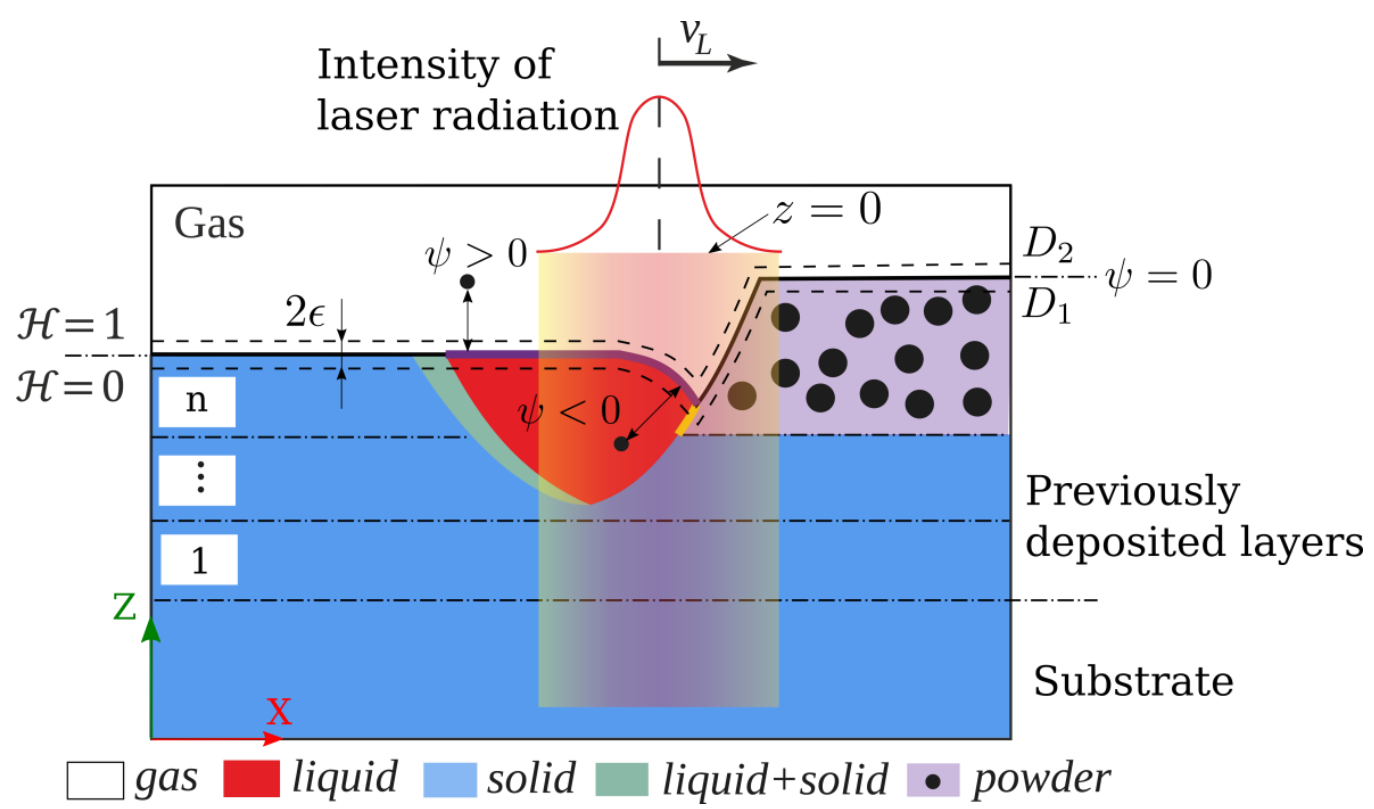

Figure 1: Schematic representation of AM by SLM as modelled using a LS method. The purple interface is between liquid ceramic and gas, whereas the yellow interface is between liquid ceramic and non-melted powder bed.

The system consists of two domains. The material domain, $D_{1}$, is separated from the gas domain, $D_{2}$, as shown in Figure 1. The boundary of these two domains is immersed in the system and a level set function $\psi$ is defined to track it. This signed distance function is taken as positive (resp. negative) in gas (resp. material) domain. A transition zone is introduced around the boundary $(\psi=0)$ with a half thickness $\epsilon$ in which the Heaviside function, $\mathcal{H}(\psi)$, evolves from 0 to 1 and keeps constant outside ( 1 in gas and 0 in material domain):

$$
\mathcal{H}(\psi)=\left\{\begin{array} { c } 
{ 0 } \\
{ \frac { 1 } { 2 } [ 1 + \frac { \psi } { \epsilon } + \frac { 1 } { \pi } \operatorname { s i n } ( \frac { \pi \psi } { \epsilon } ) ] } \\
{ 1 }
\end{array} \quad \text { if } \left\{\begin{array}{c}
\psi<-\epsilon \\
|\psi| \leq \epsilon \\
\psi>\epsilon
\end{array}\right.\right.
$$

Contrary to domain $D_{2}$, which is made of a single gas phase, the material domain, $D_{1}$, is multiphase. Its average volumetric properties $\langle\chi\rangle^{D_{1}}$ are expressed by: 


$$
\langle\chi\rangle^{D_{1}}=\sum_{\varphi} g_{D_{1}}^{\varphi} \chi^{\varphi}
$$

where $g_{D_{1}}^{\varphi}$ is the volume fraction of phase $\varphi$ in the domain $D_{1}$ and $\chi^{\varphi}$ is the intrinsic property of phase $\varphi$. The average system properties $\{\chi\}$ are computed over the two-domain system by using the Heaviside function [27][28]:

$$
\{\chi\}=\mathcal{H}\langle\chi\rangle^{D_{2}}+(1-\mathcal{H})\langle\chi\rangle^{D_{1}}
$$

This averaged approach is used to define a set of thermo-physical properties including density, enthalpy, thermal conductivity and viscosity. It aims at defining thereafter a set of equations averaged over the whole system corresponding to energy, momentum and mass conservation.

\subsection{Energy conservation}

The transient heat transfer equation, taking into account convection and diffusion phenomena, is expressed as follows:

$$
\frac{\partial\{\rho h\}}{\partial t}+\nabla \cdot\{\rho h \boldsymbol{u}\}-\nabla \cdot\{\lambda \nabla T\}=\dot{q}_{L}-\dot{q}_{r}
$$

where average quantities are the temperature, $T$, the density, $\rho$, the specific enthalpy, $h$, and the thermal conductivity, $\lambda$. The convection velocity, $\boldsymbol{u}$, is the solution of the Navier-Stokes equation (see Section 2.3). The weak form of the non-linear Eq. (4) is solved on the whole system by use of a preexisting FE solver [29]. This solver - not presented here - is based on a non-linear temperature resolution using the average enthalpy which is provided by tabulated phase transformation paths and tabulated phase properties. This general description of phase transformation allows studying any phase change during heating and cooling in the case where the phase fractions $g_{D_{1}}^{\varphi}$ depend on the temperature only. As regards evaporation, the phenomenon is less important with ceramics as compared to the processing of metals, due to high boiling points $\left(2977^{\circ} \mathrm{C}\right.$ for alumina). Hence, the liquid/gas phase transformation is actually not taken into account in the present model. This is validated a posteriori by observing that the maximum temperature found in the following simulations is below the boiling point. The next two paragraphs detail the specific expression of the right hand side terms of Eq. (4).

\subsubsection{Surface heat loss}

In the context of the level set formulation, the heat loss by radiation at the surface of the material domain $(\psi=0)$ is taken into account by introducing a volume heat extraction rate:

$$
\dot{q}_{r}=\delta \varepsilon_{r} \sigma\left(T^{4}-T_{0}^{4}\right)
$$


where $\sigma$ is the Stefan-Boltzmann constant, $T_{0}$ is the environment temperature and $\varepsilon_{r}$ is the emissivity of the powder bed surface [30]. Here the Continuous Surface Force (CSF) method [31] is used to transform the surface heat loss into a volume heat extraction by multiplying it by the Dirac function $\delta(=\partial \mathcal{H} / \partial \psi)$.

\subsubsection{Laser interaction}

Because of the low absorption of ceramic with respect to the $1064 \mathrm{~nm} \mathrm{Nd:YAG} \mathrm{laser}$ radiation used for processing, the volume heat source $\dot{q}_{L}$ is modelled based on the BeerLambert law [19]:

$$
\dot{q}_{L}(r, z)=\alpha(1-R) \frac{2 P_{L}}{\pi r_{i n t}^{2}} \exp \left(-\frac{2 r^{2}}{r_{i n t}^{2}}\right) \exp \left(-\int_{0}^{z} \alpha d l\right)
$$

Here $R$ is the reflection coefficient at the powder bed surface, $P_{L}$ is the nominal laser power, $\alpha$ is the local absorption coefficient, $r$ is the radial distance to the laser beam axis. In this model, the absorption coefficient appears in the first and last terms of the right-hand side, the latter representing the attenuation in depth. Note that due to energy dispersion in the powder layer, the interaction radius $r_{i n t}$ is increased compared with the nominal beam radius $r_{L}$ [12]. In the numerical implementation, the heat source is imposed in the region $r \in\left[0,1.5 r_{\text {int }}\right]$, corresponding to $98.9 \%$ of the nominal power. Normalization is achieved to deliver the nominal net global power $P_{L}(1-R)$.
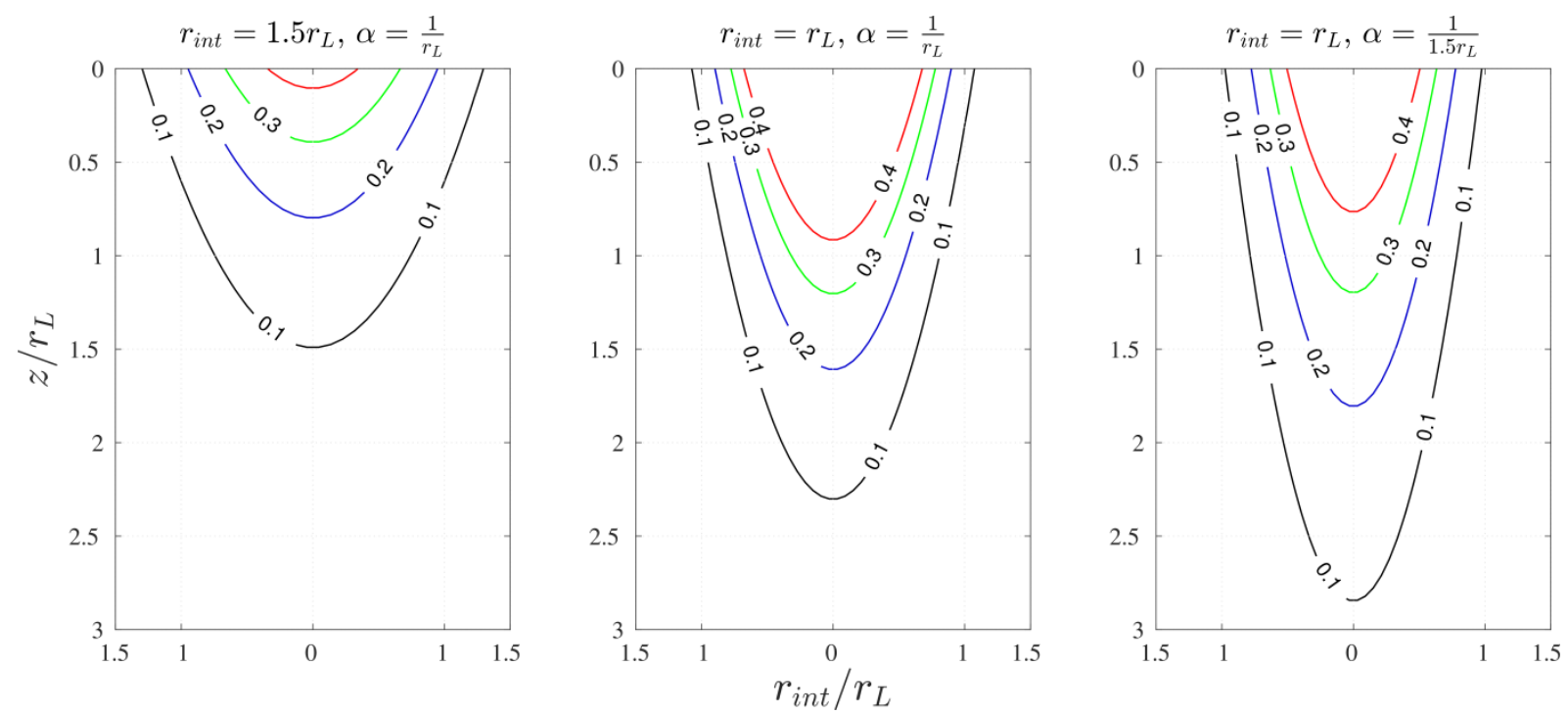

Figure 2: Energy distribution with different values of $r_{\text {int }}$ and $\alpha$ (Eq. (5)) under the same $R$ and $P_{L}$, isocontours corresponding to $\dot{q}_{L}=0.1,0.2,0.3$ and $0.4 \dot{q}_{L \max }$, where $\dot{q}_{L \max }=$ $(1-R) \frac{2 P_{L}}{\pi r_{L}^{3}}$. 
The energy distribution with different values of $r_{i n t}$ and $\alpha$ under the same $R$ and $P_{L}$ is illustrated in Figure 2. The increase of $r_{i n t}$ always leads to shallower isocontours, while it becomes narrower (e.g. isocontour 0.4) near the center but wider (e.g. isocontour 0.1) far away from the center. On the other hand, the decrease of $\alpha$ always results in narrower isocontours, while its influence on the isocontour depth is not monotonous. With smaller values for $\alpha$ coefficient, the region with high energy becomes shallower (e.g. isocontour 0.4) and the one with low energy becomes deeper (e.g. isocontours 0.1 and 0.2 ).

\subsection{Momentum conservation}

The velocity is computed in the whole system by the resolution of the average momentum conservation equation:

$$
\{\rho\}\left(\frac{\partial\{\boldsymbol{u}\}}{\partial t}+(\{\boldsymbol{u}\} \cdot \nabla)\{\boldsymbol{u}\}\right)-\boldsymbol{\nabla} \cdot\{\underline{\underline{\sigma}}\}=\boldsymbol{f}_{s}+\boldsymbol{f}_{m}+\{\rho\} \boldsymbol{g}
$$

where $\boldsymbol{u}$ is the velocity, $\underline{\underline{\boldsymbol{\sigma}}}$ is the stress tensor, $\boldsymbol{g}$ is the gravity vector, $\boldsymbol{f}_{s}$ and $\boldsymbol{f}_{m}$ are the volume forces corresponding to surface tension and Marangoni forces, respectively. These contributions are detailed hereafter.

\subsubsection{Behavior law}

As the powder is described as a continuous medium, the apparent density of the material domain $D_{1}$ varies strongly during SLM. As a consequence, shrinkage takes place during consolidation from powder to compact material. Thus a compressible Newtonian constitutive law is used in the whole system including material and gas domains:

$$
\begin{aligned}
\underline{\underline{\boldsymbol{\sigma}}}=\underline{\underline{\boldsymbol{s}}}-p \underline{\underline{\boldsymbol{I}}} \underline{\underline{\boldsymbol{s}}}=2 \mu\left(\underline{\underline{\dot{\boldsymbol{\epsilon}}}}-\frac{1}{3} \operatorname{tr}(\underline{\underline{\dot{\boldsymbol{\epsilon}}}}) \underline{\underline{\boldsymbol{I}}}\right) \\
\operatorname{tr}(\underline{\underline{\dot{\boldsymbol{\epsilon}}}})=\boldsymbol{\nabla} \cdot\{\boldsymbol{u}\}
\end{aligned}
$$

where $p$ is the pressure, $\mu$ is the dynamic viscosity, $\underline{\underline{\boldsymbol{s}}}$ is the deviatoric part of $\underline{\underline{\boldsymbol{\sigma}}}, \underline{\boldsymbol{I}}$ is the identity tensor and $\underline{\underline{\boldsymbol{\epsilon}}}$ is the strain rate tensor. Here $\boldsymbol{\nabla} \cdot\{\boldsymbol{u}\}$ is a term related to the shrinkage rate deduced from the mass conservation equation as described below.

\subsubsection{Surface forces implemented as volume forces}

Both the surface tension and Marangoni force are imposed at the gas/liquid and powder/liquid interface, shown as the purple and yellow curves in Figure 1, respectively. As these interfaces are immersed in the system, the surface forces are multiplied by a Dirac 
function $\delta_{S T}$ to transform them into volume forces. Note that $\delta_{S T}$ differs from the previously defined $\delta$ function, as it is computed by another level set associated to gas/liquid (purple curve) and powder/liquid (yellow curve) interfaces. The volume force associated with surface tension can then be expressed as:

$$
\boldsymbol{f}_{S}=\delta_{S T} \gamma \kappa \boldsymbol{n}
$$

where $\gamma$ is the surface tension coefficient, $\kappa=-\boldsymbol{\nabla} \cdot \boldsymbol{n}$ is the average curvature and $\boldsymbol{n}$ is the unit vector normal to the interface. In addition to the surface tension force which is normal to the interface, the volume force associated with the Marangoni surface force is expressed by:

$$
\boldsymbol{f}_{m}=\delta_{S T} \frac{\partial \gamma}{\partial T} \nabla_{\boldsymbol{s}} T
$$

where $\nabla_{s} T=\nabla T-(\nabla T \cdot \boldsymbol{n}) \boldsymbol{n}$ is the surface temperature gradient.

In view of FE resolution, the weak form of Eq. (7) can be established. Combined with Eqs. (8), (10) and (11) it has the following expression:

$$
\begin{gathered}
\int_{\Omega} \rho \frac{\partial \boldsymbol{u}}{\partial t} \cdot \boldsymbol{w} d \Omega+\int_{\Omega} \rho(\underline{\underline{\nabla \boldsymbol{u}}} \cdot \boldsymbol{u}) \cdot \boldsymbol{w} d \Omega+2 \int_{\Omega} \mu \underline{\underline{\dot{\boldsymbol{\epsilon}}}}(\boldsymbol{u}): \underline{\underline{\dot{\boldsymbol{\epsilon}}}}(\boldsymbol{w}) d \Omega \\
-\frac{2}{3} \int_{\Omega} \mu(\nabla \cdot \boldsymbol{u})(\nabla \cdot \boldsymbol{w}) d \Omega-\int_{\Omega} p \nabla \cdot \boldsymbol{w} d \Omega \\
=\int_{\Omega} \delta_{S T} \gamma\left[(\kappa \boldsymbol{n})^{-} \cdot \boldsymbol{w}-\Delta t\left(\underline{\underline{\nabla \boldsymbol{u}}}: \underline{\underline{\nabla \boldsymbol{w}}}-\left(\underline{\underline{\nabla \boldsymbol{u}}} \cdot \boldsymbol{n}^{-}\right) \cdot\left(\underline{\underline{\nabla \boldsymbol{w}}} \cdot \boldsymbol{n}^{-}\right)\right)\right] d \Omega \\
+\int_{\Omega} \delta_{S T} \frac{\partial \gamma}{\partial T}\left(\nabla T-\left(\nabla T \cdot \boldsymbol{n}^{-}\right) \boldsymbol{n}^{-}\right) d \Omega+\int_{\Omega} \rho \boldsymbol{g} \cdot \boldsymbol{w} d \Omega
\end{gathered}
$$

Here, braces are omitted for simplification and $\boldsymbol{w}$ denotes any vector test function. Note that the surface tension term is implemented with a semi-implicit formulation, as detailed in [19], providing a more stable numerical resolution.

\subsection{Mass conservation}

The average momentum conservation is coupled with the average mass conservation expressed by the divergence of the velocity field [19]:

$$
\boldsymbol{\nabla} \cdot\{\boldsymbol{u}\}=-\frac{(1-\mathcal{H})}{\langle\rho\rangle^{D_{1}}}\left(\frac{\partial\langle\rho\rangle^{D_{1}}}{\partial t}+\nabla\langle\rho\rangle^{D_{1}} \cdot\langle\boldsymbol{u}\rangle^{D_{1}}\right)-\frac{1}{4\langle\rho\rangle^{D_{1}}} \frac{\partial\langle\rho\rangle^{D_{1}}}{\partial t} \cos ^{2}\left(\frac{\pi \psi}{2 \epsilon}\right)
$$

Note that it vanishes in the gas domain $D_{2}$. The weak form of this equation is discretized using FE and solved together with Eq. (12) in a mixed velocity/pressure formulation. The obtained velocity is then used to update the gas/material interface by the transport of the level set function $\psi$ : 


$$
\frac{d \psi}{d t}=\frac{\partial \psi}{\partial t}+\boldsymbol{u}^{L S} \cdot \nabla \psi=0
$$

where $\boldsymbol{u}^{L S}$ denotes the velocity at the interface between the two domains tracked by the LS method. However, the update of the level set function $\psi$ does not guarantee the Eikonal property, $\|\nabla \psi\|=1$. In fact, this resolution is only valid for the interface $\psi=0$. Therefore, the signed distance function $\psi$ is recomputed after the resolution of Eq. (14) by a geometrical method [32] with respect to the updated position $\psi=0$.

\subsection{Mesh adaptation}

In order to capture the evolving gas/material interface represented by $\psi=0$, dynamic mesh adaptation is required. In the present model, an anisotropic mesh is used with a local size defined by a suitable metric tensor computed at each mesh node. The latter enables to follow precisely the gas/material interface as well as the evolution of the thermophysical variables (e.g. the liquid fraction). The mesh metric tensor is computed based on an error estimation combining several calculated fields [33], particularly density, liquid fraction, temperature and heat source distribution. This method refines the regions in which the second derivative (Hessian matrix) of the selected fields is high. For instance, by tracking the density field, the gas/material interface can be followed. In addition, the melt pool boundary can be refined by tracking the liquid fraction. The advantage of this method is that it minimizes the interpolation error of tracked variables while keeping a stable number of elements. Its efficiency will be shown in the next section.

\section{Application to SLM numerical modelling}

\subsection{Simulation configuration}

The dimension of the whole simulation system in present applications is $3 \times 0.5 \times 1.1 \mathrm{~mm}^{3}$ as illustrated in Figure 3. The bottom part corresponds to the substrate (grey color) with a height of $1 \mathrm{~mm}$. A layer of powder (red color) with a thickness of $30 \mu \mathrm{m}$ and porosity fraction of $50 \%$ is initially present on the substrate and the rest is gas (light blue). The simulations are restricted to a single pass developed in the $X$ direction. This configuration is assimilated to the first passes of the first layer at the beginning of process when the substrate and powder are still at room temperature. To apply the model to a representative domain during construction, more complex initial temperature should be considered in order to account for the residual heat. In addition, the current substrate should be replaced by the previously consolidated part. The material is considered as pure alumina. A laser beam with an effective power $P_{L}(1-$ 
$R)=80 \mathrm{~W}$ and an effective radius $r_{\text {int }}=35 \mu \mathrm{m}$ is applied. It moves from position $S$ with coordinates $\left(X_{S}, Y_{S}\right)=(0.2,0.25) \mathrm{mm}$ to position $E$ with coordinates $\left(X_{E}, Y_{E}\right)=(2.8,0.25) \mathrm{mm}$ at a constant scanning speed. At the beginning, the heat source is imposed progressively from $20 \%$ to $100 \%$ of effective power from $X_{S}$ to $X_{S}+2 r_{i n t}$. At the end, the heat source is switched off but the simulation continues until all the liquid is solidified. The initial and ambient temperatures are $20{ }^{\circ} \mathrm{C}$ in the whole system. Boundary conditions for thermal and fluid mechanical resolutions are detailed in Table 1.

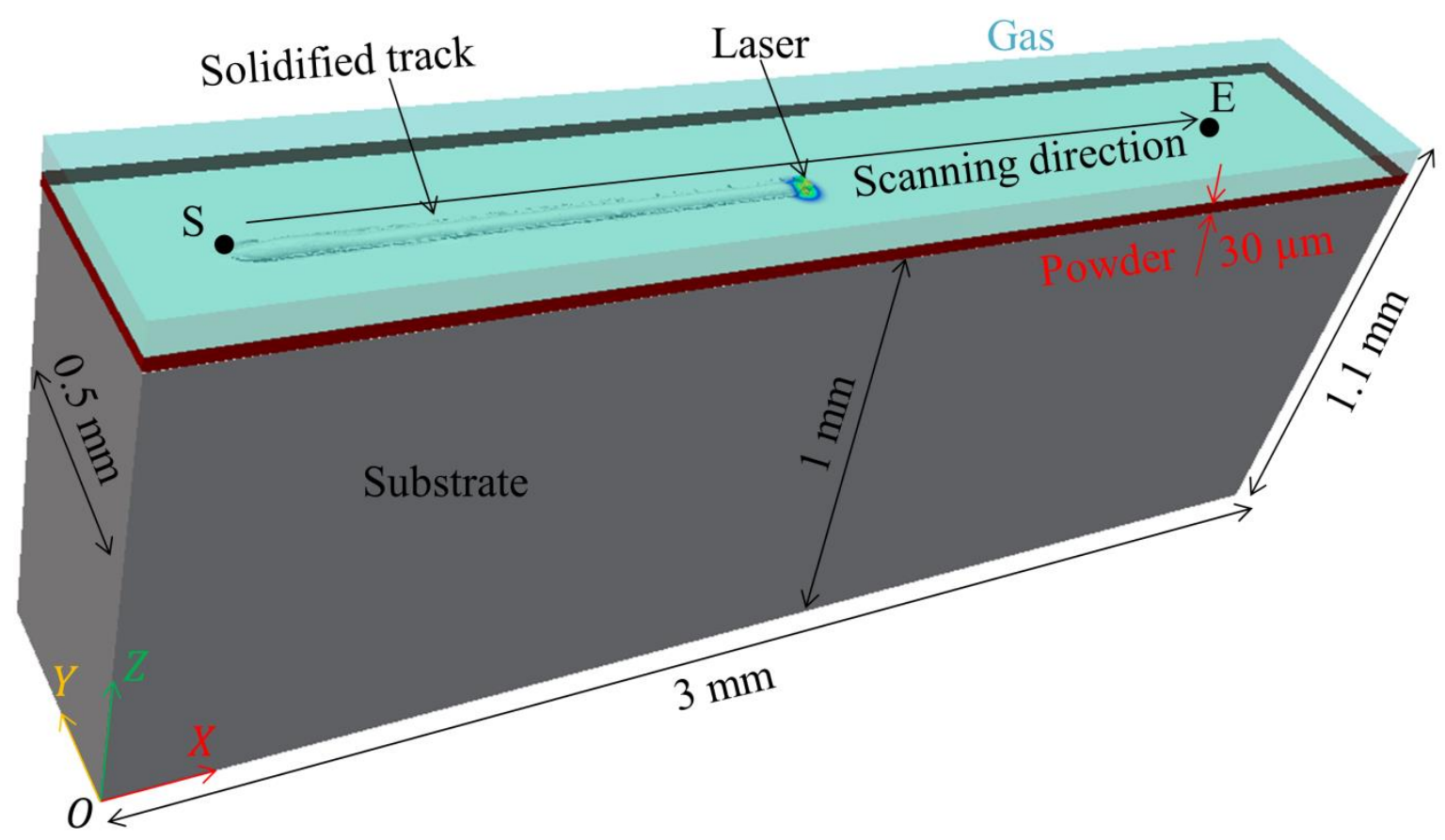

Figure 3: Configuration for unidirectional single pass simulation. A layer of powder (red) with $30 \mu \mathrm{m}$ thickness lies at the top of a substrate (grey) with $1 \mathrm{~mm}$ height. The top gas domain (light blue) is displayed with a transparent color in order to show the scanning trajectory and the solidified track on the substrate.

\begin{tabular}{|c|c|c|}
\hline & Heat transfer & Fluid flow \\
\hline Bottom & \multirow{2}{*}{ Fourier condition with heat transfer coefficient $h_{T}=40 \mathrm{~W} \mathrm{~m}^{-2} \mathrm{~K}^{-1}$} & $\boldsymbol{u}=0$ \\
\hline 4 lateral faces & & $\boldsymbol{u} \cdot \boldsymbol{n}=0$ \\
\hline Top & Adiabatic condition & Free \\
\hline
\end{tabular}

Table 1: Boundary conditions for heat transfer and fluid flow resolutions. 


\begin{tabular}{ccccc}
\hline Properties & Symbol & Value & Unit & Ref. \\
\hline Alumina density & $\rho^{a}$ & 3800 & $\mathrm{~kg} \mathrm{~m}^{-3}$ & {$[34]$} \\
Gas density & $\rho^{g}$ & 1.3 & $\mathrm{~kg} \mathrm{~m}^{-3}$ & {$[35]$} \\
Specific enthalpy of alumina & $h^{a}$ & Fig.5 (a) in Ref [19] & $\mathrm{J} \mathrm{kg}^{-1}$ & {$[36]$} \\
Specific heat of gas & $C_{p}^{g}$ & 1000 & $\mathrm{~J} \mathrm{~kg}^{-1} \mathrm{~K}^{-1}$ & {$[35]$} \\
Thermal conductivity of & $\lambda^{a}$ & $5.5+34.5 \exp (-0.0033 T)$ & $\mathrm{W} \mathrm{m}^{-1} \mathrm{~K}^{-1}$ & {$[37]$} \\
alumina & $\lambda^{g}$ & $T \in[25,1300]{ }^{\circ} \mathrm{C}$ & $\mathrm{W} \mathrm{m}^{-1} \mathrm{~K}^{-1}$ & {$[35]$} \\
Thermal conductivity of gas & $\mu^{g}$ & 0.024 & $\mathrm{~Pa} \mathrm{~s}^{-1}$ & {$[35]$} \\
Gas viscosity & $\langle\alpha\rangle^{Z_{1}}$ & $4.4 \times 10^{-5}$ & $\mathrm{~mm}^{-1}$ & {$[19]$} \\
Powder absorption & 3 & $\mathrm{~mm}^{-1}$ & {$[19]$} \\
Dense matter absorption & $\langle\alpha\rangle^{Z_{2}}$ & 3 & & \\
\hline
\end{tabular}

Table 2: Material properties of alumina and gas.

\begin{tabular}{c|c|c|c|c|c|c}
\hline \multicolumn{2}{|c|}{ Parameters } & \multicolumn{5}{|c}{ Case } \\
\hline Symbol & Unit & $\# 1$ (reference) & $\# 2$ & $\# 3$ & $\# 4$ & $\# 5$ \\
\hline$\frac{\partial \gamma}{\partial T}$ & $\times 10^{-5} \mathrm{~N} \mathrm{~m}^{-1} \mathrm{~K}^{-1}$ & -8.2 & $-\mathbf{4 8}$ & $+\mathbf{4 8}$ & \multicolumn{2}{|c}{$\# 1$} \\
\hline$v_{L}$ & $\mathrm{~mm} \mathrm{~s}^{-1}$ & 300 & \multicolumn{2}{|c|}{$\# 1$} & $\mathbf{5 0 0}$ & $\# 1$ \\
\hline$r_{i n t}$ & $\mu \mathrm{m}$ & 35 & \multicolumn{2}{|c}{$\# 1$} & $\mathbf{5 0}$ \\
\hline
\end{tabular}

Table 3: Set of process and physical parameters used for the simulation cases. Values in bold red color highlight the modified parameters with respect to the reference case.

Principal material properties are provided in Table 2 and more details can be found in Ref [19]. Specific additional comments are given hereafter for the viscosity and surface tension of liquid alumina, which are both considered as temperature dependent. First, the expression of the dynamic viscosity (in $\mathrm{Pa} \mathrm{s}$ ) as a function of temperature (in $\mathrm{K}$ ) is obtained by fitting the results of Langstaff et al. [38] with an Arrhenius law:

$$
\mu=2.15 \times 10^{-3} \exp \left(\frac{57685}{R T}\right)
$$

where $R$ is the gas constant: $8.314 \mathrm{~J} \mathrm{~mol}^{-1} \mathrm{~K}^{-1}$. Viscosity values are found between $42.4 \mathrm{mPa} \mathrm{s}$ at the melting temperature $T_{m}=2054{ }^{\circ} \mathrm{C}$ and $31.9 \mathrm{mPa}$ s at $2300{ }^{\circ} \mathrm{C}$. Secondly, the surface tension is assumed to vary linearly with temperature:

$$
\gamma=\gamma_{m}+\frac{\partial \gamma}{\partial T}\left(T-T_{m}\right)
$$

where $\gamma_{m}$ is the surface tension at melting point $T_{m}$. The temperature slope of the surface tension coefficient, $\partial \gamma / \partial T$, is taken from the literature where different measurement methods yield values varying between $-4.8 \times 10^{-4}$ and $-6 \times 10^{-5} \mathrm{~N} \mathrm{~m}^{-1} \mathrm{~K}^{-1}$ [39]. This variation domain being large, it is proposed here to develop SLM simulations in order to show firstly the influence of this coefficient on fluid dynamics and track formation. The parameters of presented cases are detailed in Table 3. The results of Paradis and Ishikawa [39] with $\gamma_{m}=$ 
$0.64 \mathrm{~N} \mathrm{~m}^{-1}$ and $\partial \gamma / \partial T=-8.2 \times 10^{-5} \mathrm{~N} \mathrm{~m}^{-1} \mathrm{~K}^{-1}$ are used as the reference case \#1. Then the extreme literature value $\partial \gamma / \partial T=-4.8 \times 10^{-4} \mathrm{~N} \mathrm{~m}^{-1} \mathrm{~K}^{-1}$ is applied in case \#2, while in case $\# 3$ an artificial positive value aims at studying the influence of the sign of $\partial \gamma / \partial T$. The effect of the scanning speed and the effective interaction radius on the temperature distribution and melt pool stability are investigated in cases \#4 and \#5. The time step is set to $2 \mu$ s for cases \#1 to \#3 and $1.5 \mu$ s for cases \#4 and \#5. The number of mesh elements increases from around 800000 to 1200000 from the beginning to the end of simulations in order to have a good representation of the track surface.

\subsection{Results and discussion}

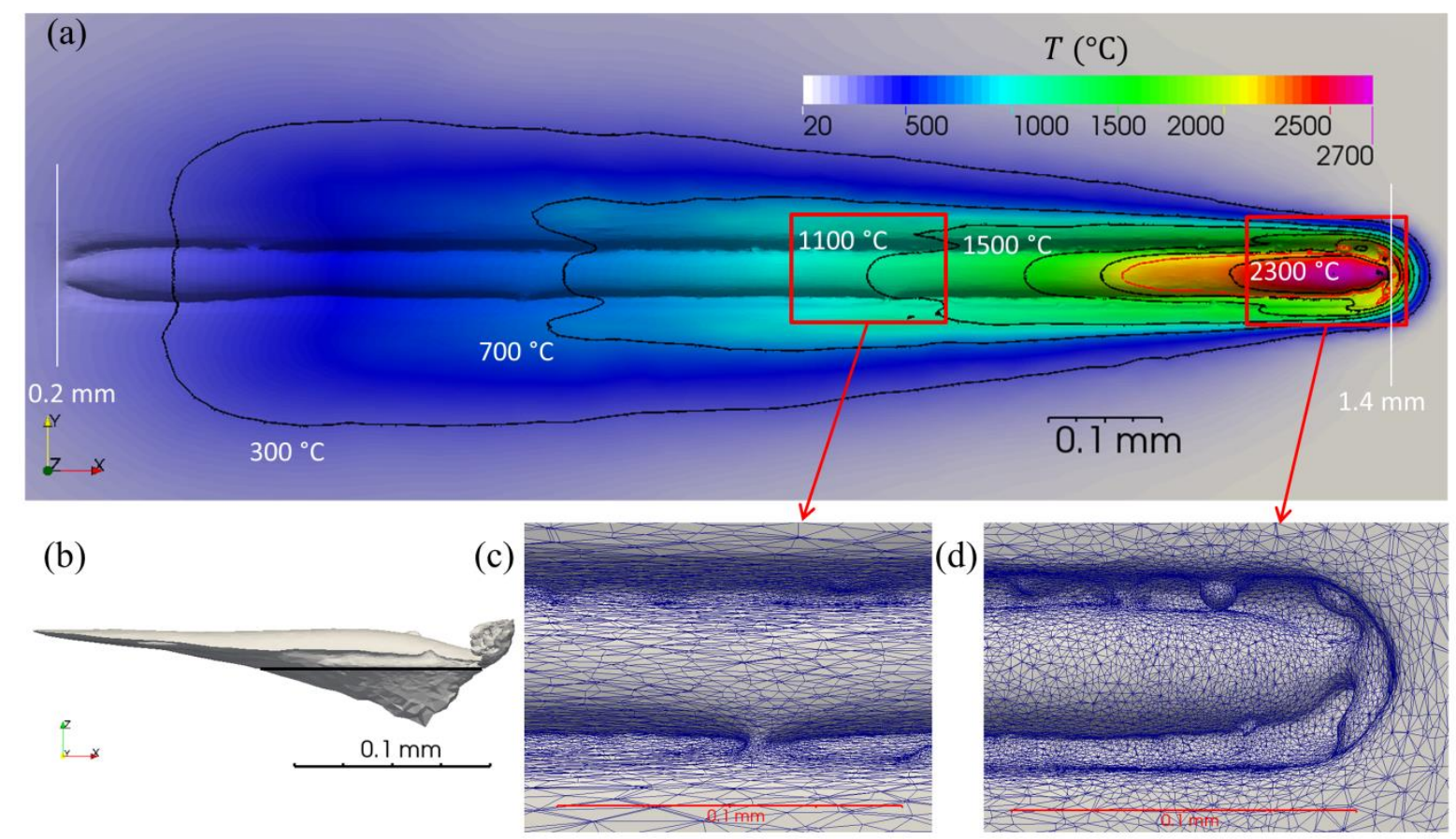

Figure 4: (a) Temperature field (top view) and isocontours (black lines) from 300 to $2300{ }^{\circ} \mathrm{C}$ with $400{ }^{\circ} \mathrm{C}$ interval. The liquidus line is also presented (red contour) for $T=2104{ }^{\circ} \mathrm{C}$; (b) melt pool shape with the surface position of substrate (black line); (c)-(d) mesh in the solidified track and at the melt pool front when the laser arrives at position $X=1.4 \mathrm{~mm}(t=4$ $\mathrm{ms}$ ) for case \#1 (Table 3). Note that the gas domain is removed in all figures so as to see the material domain. (see attached video SingleTrack_Case_1)

Temperature field with isocontours of the reference case \#1 is shown in Figure 4 (a) when the laser reaches position $X=1.4 \mathrm{~mm}$ (at time $t=4 \mathrm{~ms}$ ), i.e. approximately at middistance of the total track length (see attached video SingleTrack_Case_1). The melt pool is 
elongated behind the laser spot with a length of about $240 \mu \mathrm{m}$. The isocontours show an evolution of the tail shape during cooling. It changes from a convex shape at high temperature to a non-convex shape at low temperature, with an enlargement into the powder bed. At high temperature, for example for $T>1100{ }^{\circ} \mathrm{C}$ in the region corresponding to the red window used to identify the Figure 4 (c), the solidified track is hotter than the powder on its lateral sides. However, when it is cooled to $700{ }^{\circ} \mathrm{C}$, the track becomes colder than the neighboring powder. This evolution is caused by the insulant effect of powder due to its lower thermal conductivity $\left(\approx 0.2 \mathrm{~W} \mathrm{~m}^{-1} \mathrm{~K}^{-1}\right)$ as a porous material $[15,35]$.

Moreover, one significant phenomenon in SLM is the high temperature gradient induced by rapid heating. Generally, the temperature gradient ahead of the laser spot is much higher than that behind it, as the heat input at front cannot be rapidly conducted and transferred. The average temperature gradient in the track just behind the melt pool is about $6 \times 10^{6} \mathrm{~K} \mathrm{~m}^{-1}$ (estimated with isocontours $T=1500{ }^{\circ} \mathrm{C}$ and $T=1900{ }^{\circ} \mathrm{C}$ ), while it is about $2 \times 10^{6} \mathrm{~K} \mathrm{~m}^{-1}$ (estimated with isocontours $T=2014{ }^{\circ} \mathrm{C}$ and $T=2300{ }^{\circ} \mathrm{C}$ ) in the melt pool, as the convection tends to homogenize the temperature field. The melt pool shape in Figure 4 (b) shows a long tail and a penetration of about $50 \mu \mathrm{m}$ in the substrate. It is largely influenced by the laser energy distribution (depending on the laser power, reflection, interaction radius and material absorption) and the scanning speed. Consequently, a desired melt pool penetration in substrate can be obtained by a good control of the process parameters. At the front of the melt pool, liquid droplets form by powder melting and spheroidization under the surface tension effect. These droplets lead to the discontinuity of the liquid zone. They fall into the melt pool under gravity and cause instabilities or wave propagation in the melt pool. This phenomenon is captured by the use of the LS method and the efficient mesh adaptation approach previously detailed. The latter is an important issue in the present FE method and the mesh size control is shown in Figure 4 (c) and (d). By tracking two solution fields (density and liquid fraction), the melt pool with high curvature is well refined, especially for the melted powder region with a minimum mesh size around $0.5 \mu \mathrm{m}$ as shown in Figure 4 (d). However, once the track is solidified, liquid fraction has no more effect on the mesh refinement and a larger mesh size is retrieved (Figure 4 (c)). In addition, elements are elongated in the $X$ direction as low density gradient variation is encountered in this direction, leading to an anisotropic mesh. 

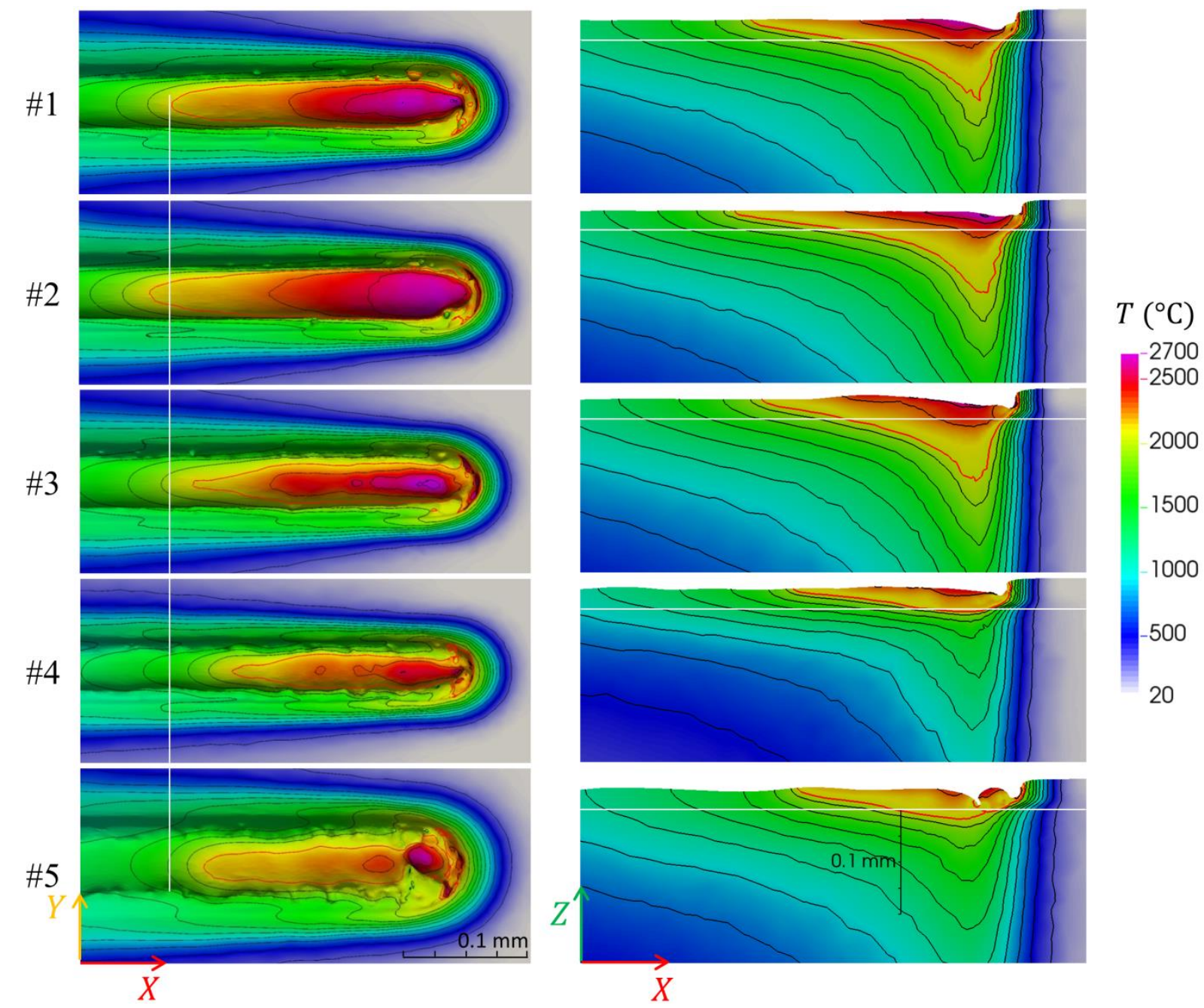

Figure 5: Top (left column) and longitudinal cut view (right column) of temperature field for cases \#1 to \#5 when the laser arrives at position $X=1.4 \mathrm{~mm}$. Temperature isocontours (black) range from 300 to $2700{ }^{\circ} \mathrm{C}$ with $200{ }^{\circ} \mathrm{C}$ interval and the red isocontour corresponds to the melt pool. The vertical white line at left is for comparing the melt pool length and the horizontal one at right indicates the surface of substrate.

The Marangoni effect on the temperature distribution can be investigated by comparing cases \#1 to \#3 in Figure 5 (laser at $X=1.4 \mathrm{~mm}$ and $t=4 \mathrm{~ms}$ ). With a higher negative value of $\partial \gamma / \partial T$ in case \#2 (Table 3), the melt pool (red color) is enlarged both in width and length. This also occurs for the temperature isocontours above the liquidus temperature. Consequently, the isocontours in the solidified track are also extended. By contrast, a positive value of $\partial \gamma / \partial T$ (case \#3) reduces the melt pool, leading to a narrower and shorter pool shape. This effect can result in the necking of isocontours, for example at $T=2300{ }^{\circ} \mathrm{C}$, which is also indicated in the longitudinal cut view. The consequence of the necking is the interruption of isocontours, such as the one corresponding to $T=2500{ }^{\circ} \mathrm{C}$. By comparing cases \#1 to \#3, it is shown that the melt pool becomes unstable when the Marangoni coefficient $\partial \gamma / \partial T$ varies 
from the negative to the positive value. However, the Marangoni effect on the depth of melt pool is not prominent for these cases \#1 to \#3.

As a second step in simulation strategy, the scanning speed has been increased to 500 $\mathrm{mm} \mathrm{s}^{-1}$. This leads to a reduction in the deposited energy and consequently to a smaller melt pool, as shown in case \#4 (Figure 5). In addition, the high scanning speed results in the discontinuity of temperature isocontours. On the other hand, with a higher interaction radius as proposed in case \#5 (Figure 5), powder is heated in a wider region. Correspondingly, more powder is melted at the melt pool front and larger droplets are formed, leading to a more unstable melt pool. In both cases \#4 and \#5, the melt pool is much shallower than that in case $\# 1$ and it can just melt the substrate surface.
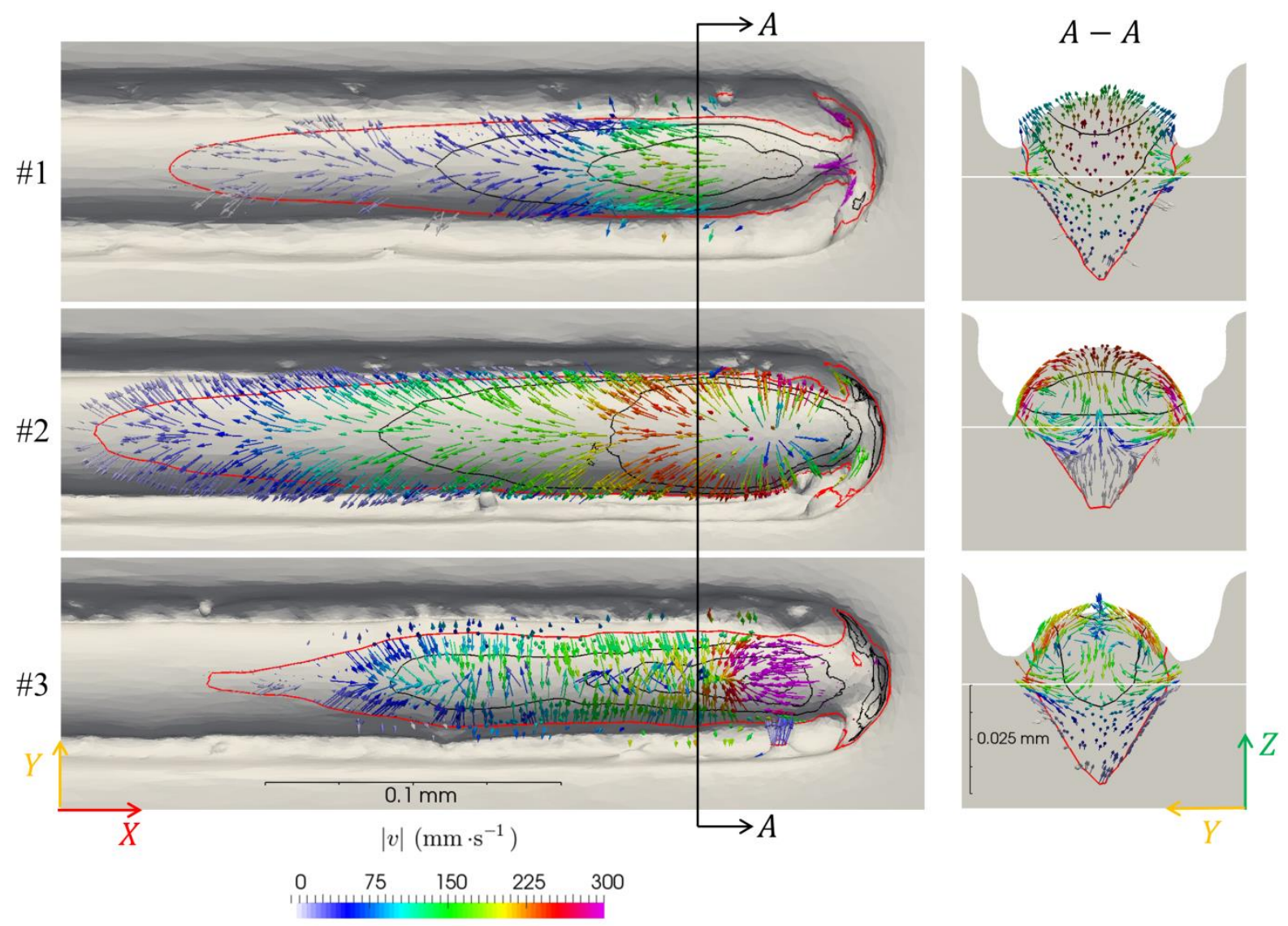

Figure 6: Velocity field (arrows colored by magnitude) at the surface of melt pool and the $A-A$ section for cases \#1 to \#3 when the laser arrives at position $X=1.4 \mathrm{~mm}$ for $t=4 \mathrm{~ms}$. Temperature isocontours are the same as in Figure 5.

The temperature distribution in the melt pool is largely influenced by the convection flow. Top and transverse cut views are presented in Figure 6. Several phenomena contribute to fluid flow. Firstly, the melted powder at the front of the melt pool forms droplets under the effect of surface tension. Discontinuous fall events of these droplets in the pool under the 
effect of gravity create waves that propagate toward the rear of the melt pool. This explains the velocity distribution for case \#1 shown in Figure 6 where the surface velocity field is essentially oriented toward the rear of the melt pool near section $A-A$ (green arrows) after the fall event. This convection flow is also affected by the Marangoni effect, leading to spreading of the melt toward the two sides of the melt pool. The latter is more evident in case \#2 when the magnitude of $\partial \gamma / \partial T$ is increased. Because $\partial \gamma / \partial T<0$, the convection flow is induced from hotter region (low surface tension) to colder region (high surface tension). This flow drives heat outwards and extends the boundary of the melt pool, as well as the temperature isocontours. The transverse cut view shows clearly that two opposite convection cells are formed. At the surface of melt pool, centrifugal flow is driven from the center (hotter region) to lateral sides (colder region), leading to a wider melt pool. In the transversal cutting plane, the flow is upward to the melt pool surface. These convection cells result in the transport of fluid and increase heat exchange, thus changing the isocontours $\left(T=2300{ }^{\circ} \mathrm{C}\right.$ and $T=$ $2500{ }^{\circ} \mathrm{C}$ ). In contrast to case \#2, a positive $\partial \gamma / \partial T$ with the same magnitude as in case \#3 shows convection flow in the opposite direction. The flow is driven from colder to hotter regions, as surface tension coefficient increases with temperature. This leads to the reduction of melt pool surface and temperature isocontours, or even discontinuity $\left(T=2500{ }^{\circ} \mathrm{C}\right)$. Two convection cells in opposite directions compared to those of case \#2 are observed in the transverse cut view. Fluid flows inward at the surface of the melt pool and downward along the longitudinal symmetry plane. However, the convection cells are limited to a weak penetration into the substrate, probably because of the relatively high viscosity of liquid alumina (compared to the viscosity of liquid metals). Consequently, the melt pool depth is almost unchanged, comparing cases \#2 and \#3 with the reference case \#1.

One of the possible consequences of the melt pool dynamics is the irregular profile of the solidified tracks, with interruption of track or balling effect. The surface heights of the final tracks in cases \#1 to \#5 are shown in Figure 7, together with isocontours. A transition region at the beginning is observed where a swelling occurs. However, the domain of interest is the quasi-steady state which follows. The regularity of the track profile is perceptible by the color difference along the track and by the discontinuity of isocontours. The high altitude regions (hills) are highlighted by small circles and the necks of isocontours are the low altitude regions (valleys). Compared to case \#1, the number of hills and valleys decreases in case \#2, meaning a more stable surface height when $\partial \gamma / \partial T$ is decreased. By contrast, the increase of $\partial \gamma / \partial T$ leads to more hills and valleys, thus a higher track irregularity in case \#3. The Marangoni effect on the track regularity can be explained by the convection flow in Figure 6 . If we consider a melt pool with two successive liquid hills and a valley between them, convection flow with negative $\partial \gamma / \partial T$ tends to drive fluid from hills to valleys, thus reducing the height difference between them. Conversely, a positive value of $\partial \gamma / \partial T$ can promote this 
difference and enhance irregularities. This means that a high magnitude negative $\partial \gamma / \partial T$ is favorable to obtain a smoother track surface, and may be helpful to avoid balling effect. In the simulation of processing stainless steel with SLM, Khairallah et al. [40] obtained the height variation of solidified track surface. Although the Marangoni effect was not taken into account, they guessed that negative $\partial \gamma / \partial T$ could smooth the track surface. This remark is confirmed by the present work. Case \#4 with increased scanning speed shows more hills and valleys, thus more surface instabilities. This reveals the possible tendency to the balling effect at high scanning speed, which is coherent with the results of $\mathrm{Gu}$ and Shen [23]. In addition, the solidified track is narrower than in case \#1. A larger track is obtained in case \#5 with a higher laser interaction radius, leading to an increase of melted powder. Its merge into the existing melt pool leads to instabilities and to a more marked irregular profile of the solidified track.

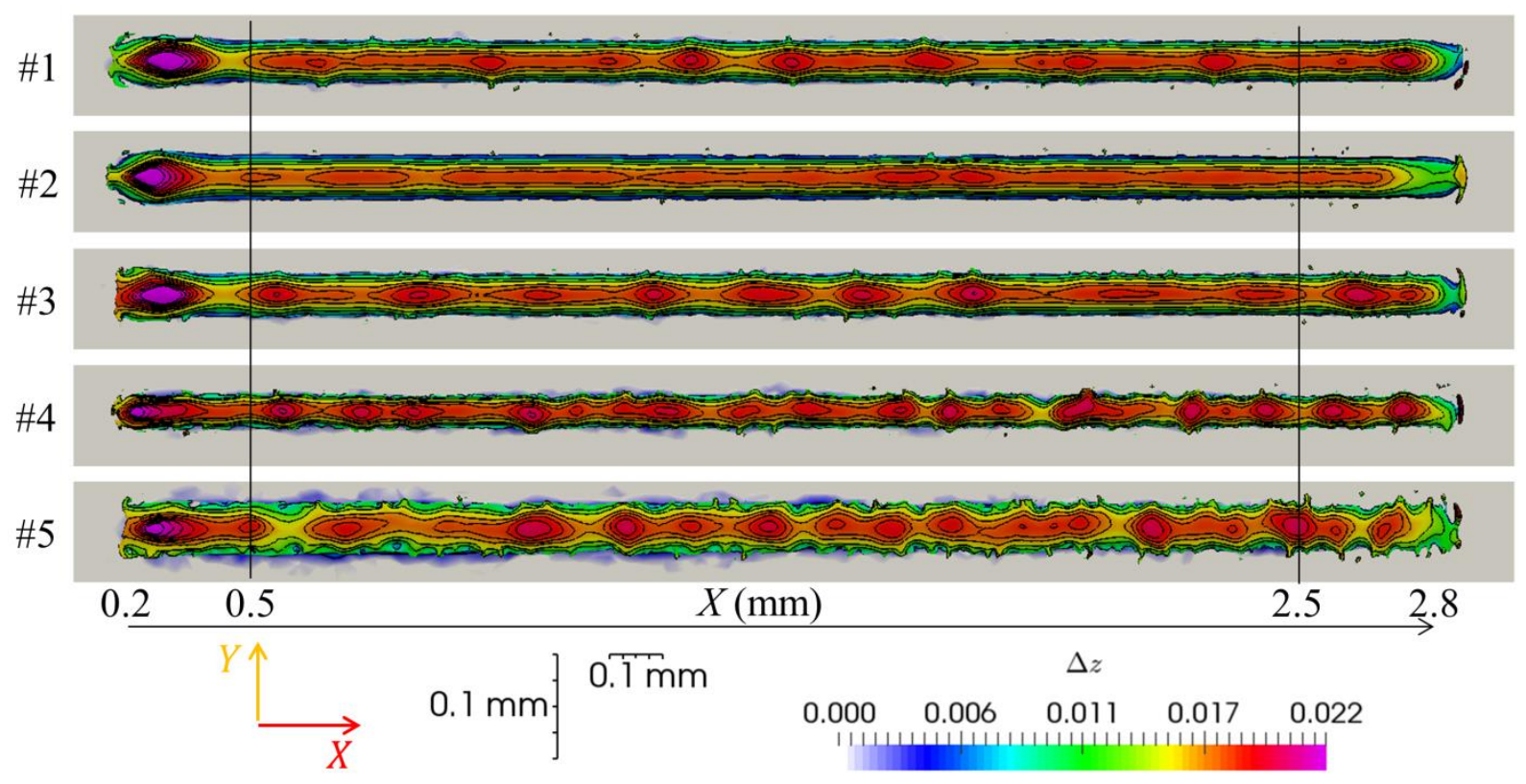

Figure 7: Height $(\mathrm{mm})$ of solidified tracks with respect to the substrate surface and isocontours for cases \#1 to \#5. The image of tracks is scaled by a factor 2 in the $Y$ direction for better visualization.

Track regularity is illustrated by the height profiles in Figure 8, taken in the longitudinal median plane of cases \#1 to \#5. Two indicators for profile regularity are selected among those adopted for roughness evaluation along a linear profile of length $L$. The first one is the total roughness $R_{t}=\max _{L} z(x)-\min _{L} z(x)$ with $x$ the spatial coordinate along the linear profile and $z(x)$ the altitude difference with respect to the average altitude over the whole profile. The second one is the average quadratic measure for roughness: $R_{q}=\sqrt{\frac{1}{L} \int_{L} Z(x)^{2} d x}$. Less height variation with a $R_{q}$ value of $0.38 \mu \mathrm{m}$ is formed in case \#2 compared to the value of 
$0.65 \mu \mathrm{m}$ in case \#1. As already noted in Figure 7, case \#3 presents a more irregular track shape with a higher $R_{q}(0.80 \mu \mathrm{m})$ and a higher $R_{t}(3.7 \mu \mathrm{m})$. In cases \#4 and \#5, a higher interaction radius or a higher scanning speed increase irregularities in track height, leading to higher values of roughness indicators.

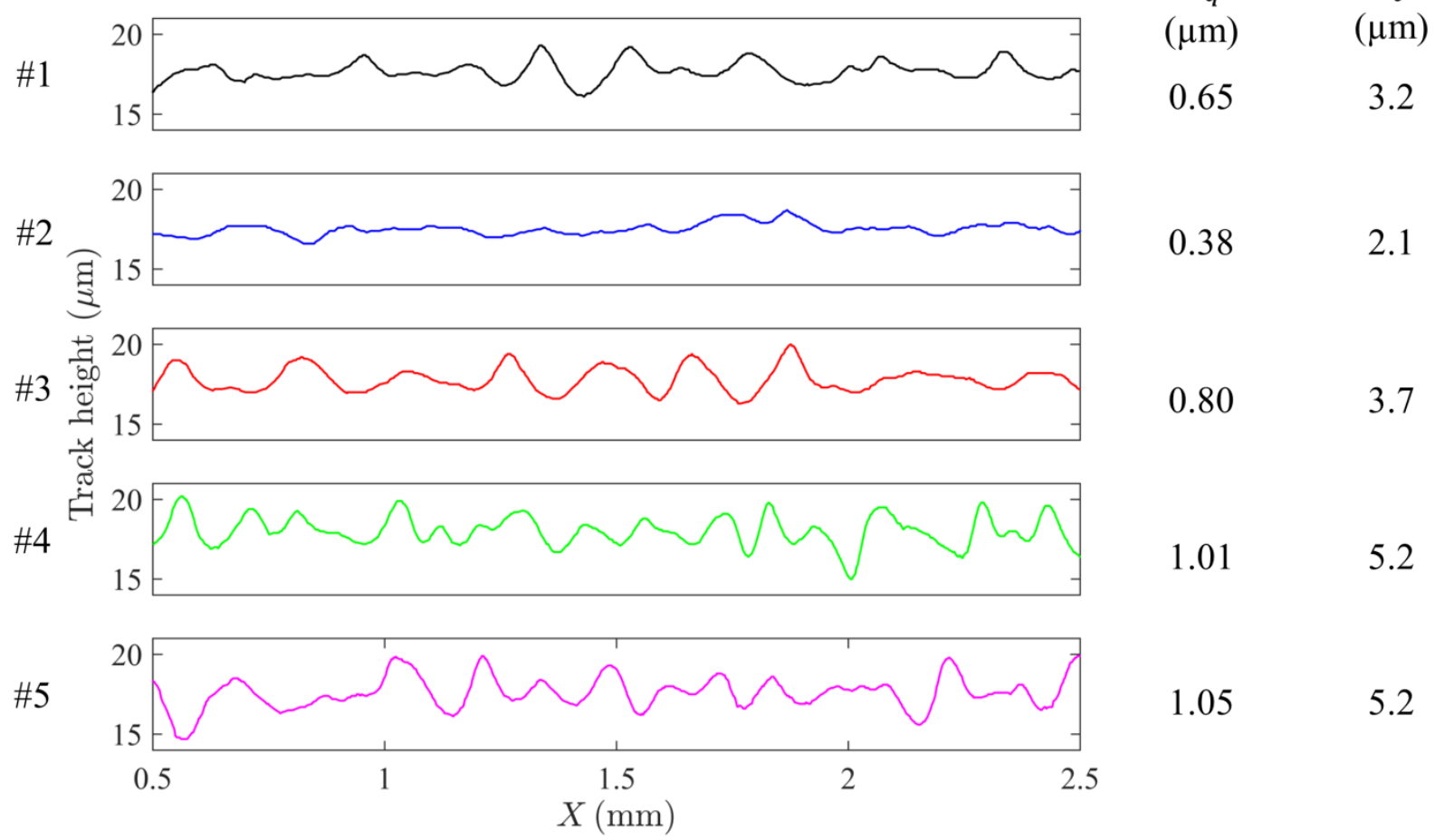

Figure 8: Height profiles in the longitudinal median plane for cases \#1 to \#5 between $X=0.5$ $\mathrm{mm}$ and $X=2.5 \mathrm{~mm}$ (i.e. the region between the two black lines in Figure 7). Indicators for track profile regularity - as defined in the text - are given on the right.

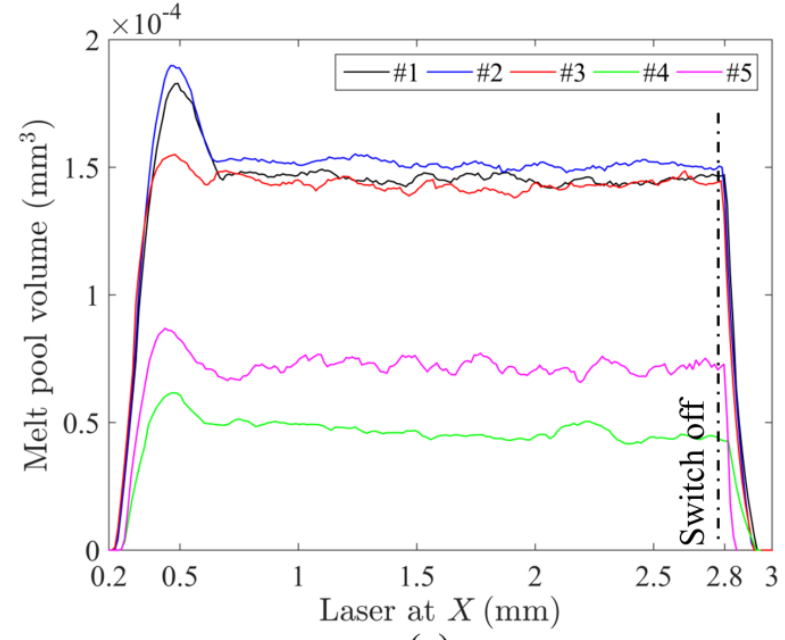

(a)

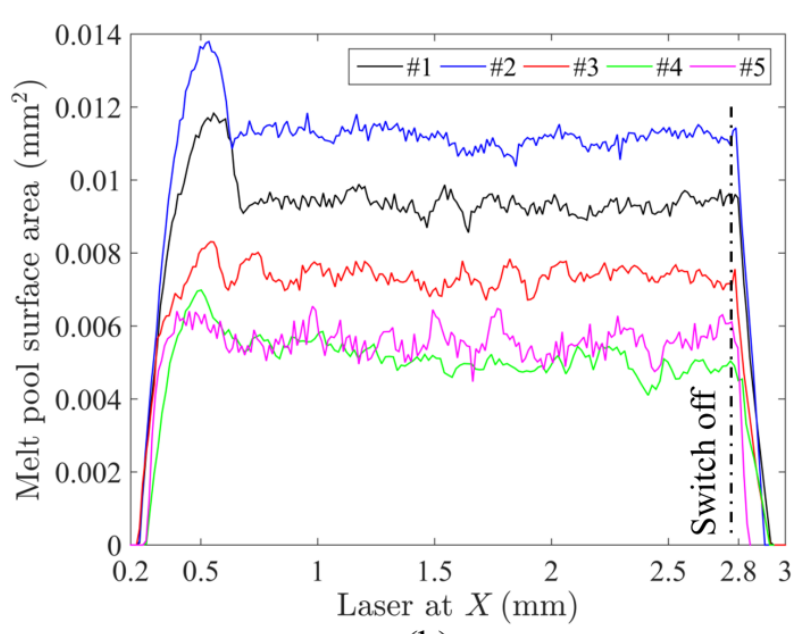

(b)

Figure 9: Evolution of (a) melt pool volume and (b) surface area for cases \#1 to \#5. 
Finally, the volume and the surface area of the melt pool (including droplets) are plotted in Figure 9 (a) and (b), in order to study its stability in cases \#1 to \#5. The transition region at the beginning of scanning is clearly shown by the peaks of melt pool volume and surface area. The stable regime begins when the laser beam arrives at position $X \sim 0.7 \mathrm{~mm}$. The melt pool volume and surface area can be compared from this position to the switch off position of the laser beam at $X=2.8 \mathrm{~mm}$. For a given case, the scattering of the surface area is much higher than that of the melt pool volume, as the whole liquid volume is conserved while this is not the case for surface area during the fall of droplets. These variations may be considered as indicators of the instability of the melt pool. Comparing with the reference case (\#1), the average volume is slightly increased in case \#2 and decreased in case \#3. However, the difference is much less significant compared with the surface area in Figure 9 (b). Due to the centrifugal surface flow along the melt pool surface in case \#2, the surface area is largely increased. By contrast, the centripetal surface flow in case \#3 leads to the reduction of the area of the melt pool surface. Both cases \#4 and \#5 have a much smaller melt pool volume than case \#1, which can be indicated by their shallower melt pool as shown in Figure 5 (longitudinal cut view). The melt pool in cases \#4 and \#5 has almost the same surface area while its volume in case \#5 is higher than that in case \#4 as the melt pool is deeper in case \#5 (Figure 5).

\section{Conclusions}

In the present approach, the effects of surface tension and Marangoni force are considered in a finite element model with level set method at the track scale, expressed in terms of continuum mechanics and heat transfer. A compressible behavior of powder is introduced to model powder shrinkage when melted. The important influence of the Marangoni effect on temperature distribution and melt pool shape is discussed by using different $\partial \gamma / \partial T$ values. It is demonstrated that a negative $\partial \gamma / \partial T$ value leads to the expansion of the melt pool, while a narrower and shorter melt pool can be obtained with a positive $\partial \gamma / \partial T$ value. Similar evolutions are observed for temperature isocontours. These observations are discussed and explained considering evolution of tangential forces induced by Marangoni effect and their direction. However, its influence on melt pool depth is not the main effect as is commonly observed for metals, due to the high viscosity of liquid ceramic. In addition, the Marangoni flow has an effect on the regularity of the profile of solidified track. A high negative value of $\partial \gamma / \partial T$ tends to smooth the surface and avoid the fragmentation of the track and associated balling effect. The scanning speed and interaction radius are also investigated. Results show that the melt pool becomes smaller and discontinuous with the increase of scanning speed or laser/material interaction radius.

Future work will focus on the comparison with experimental observations currently in progress and the quantitative validation of the present model. Then the construction of process 
map under different working conditions with different track morphologies will be carried out to better control the process. Moreover, the integration of solid mechanics should complete this model by predicting stresses in the freshly solidified track and in its vicinity, which is important regarding the definition of process windows to avoid cracking during construction.

\section{Acknowledgement}

This work has been conducted within the framework of the CEFALE project, part of the ACLAME program funded by the Institute CARNOT M.I.N.E.S (Paris, FR). The authors would like to thank Liliana Moniz Da Silva Sancho, Christophe Colin, Jean-Dominique Bartout and Marie-Hélène Berger from the MINES ParisTech, Centre des Matériaux (Evry, FR) for information and discussion regarding AM by SLM applied to ceramic materials. 


\section{References}

[1] B. Berman, 3-D printing: The new industrial revolution, Bus. Horiz., vol. 55 (2012) $155-162$.

[2] E. O. Olakanmi, R. F. Cochrane and K. W. Dalgarno, A review on selective laser sintering/melting (SLS/SLM) of aluminium alloy powders: Processing, microstructure, and properties, Prog. Mater. Sci. 74 (2015) 401-477.

[3] M. Akita, Y. Uematsu, T. Kakiuchi, M. Nakajima and R. Kawaguchi, Defect-dominated fatigue behavior in type 630 stainless steel fabricated by selective laser melting, Mater. Sci. Eng. A 666 (2016) 19-26.

[4] Y. J. Liu, S. J. Li, H. L. Wang, W. T. Hou, Y. L. Hao, R. Yang, T. B. Sercombe and L. C. Zhang, Microstructure, defects and mechanical behavior of beta-type titanium porous structures manufactured by electron beam melting and selective laser melting, Acta Mater. 113 (2016) 56-67.

[5] H. Schwab, F. Palm, U. Kühn and J. Eckert, Microstructure and mechanical properties of the near-beta titanium alloy Ti-5553 processed by selective laser melting, Mater. Des. 105 (2016) 75-80.

[6] M. Averyanova, P. Bertrand and B. Verquin, Manufacture of Co-Cr dental crowns and bridges by selective laser Melting technology, Virtual Phys. Prototyp. 6 (2011) 179-185.

[7] P. Wei, Z. Wei, Z. Chen, J. Du, Y. He, J. Li and Y. Zhou, The AlSi10Mg samples produced by selective laser melting: single track, densification, microstructure and mechanical behavior, App. Surf. Sci. 48 (2017) 38-50.

[8] Y.-C. Hagedorn, J. Wilkes, W. Meiners, K. Wissenbach and R. Poprawe, Net shaped high performance oxide ceramic parts by selective laser melting, Phys. Procedia 5 (2010) 587-594.

[9] J. Wilkes, Y.-C. Hagedorn, W. Meiners and K. Wissenbach, Additive manufacturing of $\mathrm{ZrO} 2-\mathrm{A} 12 \mathrm{O} 3$ ceramic components by selective laser melting, Rapid Prototyp. J. 19 (2013) 51-57.

[10] S. L. Sing, W. Y. Yeong, F. E. Wiria, B. Y. Tay, Z. Zhao, L. Zhao, Z. Tian and S. Yang, Direct selective laser sintering and melting of ceramics: a review, Rapid Prototyp. J. 23 (2017) 611-623.

[11] M. X. Gan and C. H. Wong, Properties of selective laser melted spodumene glassceramic, J. Euro. Ceram. Soc. 37 (2017) 4147-4154.

[12] A. V. Gusarov and J.-P. Kruth, Modelling of radiation transfer in metallic powders at laser treatment, Int. J. Heat Mass Transf. 48 (2005) 3423-3434. 
[13] X. C. Wang and J.-P. Kruth, A simulation model for direct selective laser sintering of metal powders, Computational Techniques for Materials, Composites and Composite Structures, Edinburg, 2000, 3423-3434.

[14] S. A. Khairallah, A. T. Anderson, A. Rubenchik and W. E. King, Laser powder-bed fusion additive manufacturing: physics of complex melt flow and formation mechanisms of pores, spatter, and denudation zones, Acta Mater. 108 (2016) 36-45.

[15] A. V. Gusarov and I. Smurov, Radiation transfer in metallic powder bed used in laser processing, J. Quant. Spectroc. Radiat. Trans. 111 (2010) 2517-2527.

[16] N. E. Hodge, R. M. Ferencz and J. M. Solberg, Implementation of a Thermomechanical Model for the Simulation of Selective Laser Melting, Comput. Mech. 54 (2014) 33-51.

[17] W. King, A. T. Anderson, R. M. Ferencz, N. E. Hodge, C. Kamath and S. A. Khairallah, Overview of modelling and simulation of metal powder bed fusion process at Lawrence Livermore National Laboratory, Mater. Sci. Technol. 31 (2015) 957-968.

[18] J. F. Li, L. Li and F. H. Stott, Comparison of volumetric and surface heating sources in the modeling of laser melting of ceramic materials, Int. J. Heat Mass Transf. 47 (2004) 1159-1174.

[19] Q. Chen, G. Guillemot, Ch-A. Gandin and M. Bellet, Three-dimensional finite element thermomechanical modeling of additive manufacturing by selective laser melting for ceramics materials, Additive Manufacturing 16 (2017) 124-137.

[20] M. Markl and C. Körner, Multi-scale modeling of powder-bed-based additive manufacturing, Ann. Rev. Mater. Res. 46 (2016) 1-34.

[21] Y. Liu, Y. Yang, S. Mai, D. Wang and C. Song, Investigation into spatter behavior during selective laser melting of AISI 316L stainless steel powder, Mater. Des. 87 (2015) 797-806.

[22] A. V. Gusarov, I. Yadroitsev, Ph. Bertrand and I. Smurov, Heat transfer modelling and stability analysis of selective laser melting, App. Surf. Sci. 254 (2007) 975-979.

[23] D. Gu and Y. Shen, Balling phenomena in direct laser sintering of stainless steel powder: Metallurgical mechanisms and control methods, Mater. Des. 30 (2009) 2903-2910.

[24] C. Chan, J. Mazumder and M. M. Chen, A two-dimensional transient model for convection in laser melted pool, Metall. Trans. A 15 (1984) 2175-2184.

[25] P. Yuan and D. Gu, Molten pool behavior and its physical mechanism during selective laser melting of TiC/AlSi10Mg nanocomposites: simulation and experiments. J. Phys. D: Appl. Phys. 48 (2015) 16. 
[26] C. Qiu, C. Panwisawas, M. Ward, H. C. Basoalto, J. W. Brooks and M. M. Attallah, On the role of melt flow into the surface structure and porosity development during selective laser melting, Acta Mater. 96 (2015) 72-79.

[27] O. Desmaison, M. Bellet and G. Guillemot, A level set approach for the simulation of the multipass hybrid laser/GMA welding process, Comput. Mater. Sci. 91 (2014) 240250.

[28] S. Chen, G. Guillemot and Ch.-A. Gandin, Three-dimensional cellular automaton-finite element modeling of solidification grain structures for arc-welding processes, Acta Mater. 115 (2016) 448-467.

[29] A. Saad, Ch.-A. Gandin and M. Bellet, Temperature-based energy solver coupled with tabulated thermodynamic properties - Application to the prediction of macrosegregation in multicomponent alloys, Comput. Mater. Sci. 99 (2015) 221-231.

[30] S. S. Sih and J. W. Barlow, The prediction of the emissivity and thermal conductivity of powder beds, Part. Sci. Technol. 22 (2010) 427-440.

[31] J. U. Brackbill, D. B. Kothe and C. Zemach, A continuum method for modeling surface tension, J. Comput. Phys. 100 (1992) 335-354.

[32] M. Shakoor, B. Scholtes, P.-O. Bouchard and M. Bernacki, An efficient and parallel level set reinitialization method - Application to micromechanics and microstructural evolutions, Appl. Math. Model. 39 (2015) 7291-7302.

[33] T. Coupez, Metric construction by length distribution tensor and edge based error for anisotropic adaptive meshing, J. Comput. Phys. 230 (2011) 2391-2405.

[34] R. Morrell, Handbook of Properties of Technical \& Engeering Ceramics. H.M.S.O., London, 1985.

[35] "Dry air properties", http://www.engineeringtoolbox.com/dry-air-properties-d_973.html. Accessed 11 July 2017.

[36] M. W. Chase, Thermochemical tables. NIST-JANAF, 1998.

[37] Y. S. Touloukian, R. K. Kirby, R. E. Taylor and T. T. R. Lee, Thermal expansion nonmetallic solids, Thermophys. Prop. Matter. 13 (1984) 176-177.

[38] D. Langstaff, M. Gunn, G. N. Greaves, A. Marsing and F. Kargl, Aerodynamic levitator furnace for measuring thermophysical properties of refractory liquids, Rev. Sci. Instrum. 84 (2013) 124901. 
[39] P.-F. Paradis and T. Ishikawa, Surface tension and viscosity measurements of liquid and undercooled alumina by containerless techniques, Jpn. Soc. App. Phys. 44 (2005) 50825085.

[40] S. A. Khairallah, A. Anderson, Mesoscopic simulation model of selective laser melting of stainless steel powder, J. Mater. Process. Technol. 214 (2014) 2027-2636. 